Original research article

\title{
$5 \%$ lidocaine medicated plasters vs. sympathetic nerve blocks as a part of multimodal treatment strategy for the management of postherpetic neuralgia: A retrospective, consecutive, case-series study
}

\author{
Małgorzata Malec-Milewska*, Bartosz Horosz, Agnieszka Sękowska, \\ Iwona Kolęda, Hanna Kucia, Dariusz Kosson
}

Pain Clinic: Department of Anesthesiology and Intensive Care, Medical Center for Postgraduate Education, Warsaw, Poland

\section{A R T I C L E I N F O}

\section{Article history:}

Received 20 November 2014

Accepted 5 January 2015

Available online 14 January 2015

Keywords:

Postherpetic neuralgia

Numeric rating scale

Sympathetic nerve block

Gabapentin

Tricyclic antidepressant

\begin{abstract}
A B S T R A C T
Introduction: 5\% lidocaine medicated plasters (5\% LMP) have been appointed as a first-line treatment for post-herpetic neuralgia (PHN), while formerly used sympathetic nerve blocks (SNBs) were recently denied their clinical efficacy. The aim of this study was to compare the results of PHN management with the use of SNBs and 5\% LMP as a first-line treatment. Material and methods: This study was designed as a retrospective, consecutive, case-series study. Data of 60 consecutive PHN patients with allodynia treated with the use of SNBs and 60 subsequent patients managed with 5\% LMP were analyzed. Pain severity after 8 weeks was assessed to recognize the results of the implemented therapy, with numeric rating scale (NRS) score $<3$ or $=3$ considered a success. Additionally, the number of pain-free patients $(\mathrm{NRS}=0)$ after 8 weeks were identified in both groups and compared.

Results: The rate of failures in SNBs and 5\% LMP group was similar (18.9\% vs. $27.1 \%$ of poor treatment results, respectively), with the average change in NRS of $5.88 \pm 2.41$ in nerve blocks and $5.01 \pm 1.67$ in lidocaine group $(p=0.02)$. Significant difference was also noted in the rates of pain-free patients: 20 patients (34.4\%) treated with SNBs and $8(13.5 \%)$ using $5 \%$ LMP were pain-free after 8 weeks of treatment.

Conclusion: It may be concluded that SNBs may still be considered useful in PHN management, as it appears that in some cases this mode of treatment may offer some advantages over 5\% LMP.
\end{abstract}

(C) 2015 Polish Neurological Society. Published by Elsevier Urban \& Partner Sp. z o.o. All rights reserved.

\footnotetext{
* Corresponding author at: Pain Clinic: Department of Anesthesiology and Intensive Care, Medical Center for Postgraduate Education, Ul. Czerniakowska 231, 00-416 Warszawa, Poland. Tel.: +48 225841 220; fax: +48 225841342.

E-mail address: lmilewski@post.pl (M. Malec-Milewska).

http://dx.doi.org/10.1016/j.pjnns.2015.01.001

0028-3843/C 2015 Polish Neurological Society. Published by Elsevier Urban \& Partner Sp. z o.o. All rights reserved.
} 


\section{Introduction}

The incidence of herpes zoster (HZ) and postherpetic neuralgia (PHN) is large enough for us to be recognized as a common clinical problem. Herpes zoster is thought to be a result of varicella zoster virus (VZV) reactivation, which resides in dorsal root ganglia (DRG) until its activation, following which it spreads peripherally causing distal neuropathy. Clinical presentation is usually unilateral and accompanied by pain in the affected area, which normally lasts for several days. In some cases pain extends beyond the acute phase and healing of skin lesions, which along with its neuropathic character is consistent with the diagnosis of postherpetic neuralgia (PHN). The incidence of PHN is 9-30\% of HZ cases, depending on the source cited, while $10 \%$ is probably the most likely average [1]. The management of both HZ and PHN is challenging, there are numerous approaches suggested which aim at reducing the time of acute phase of the disease and pain severity, as well as the risk of PHN. The reason for various approaches proposed in the past is obviously its complex pathophysiology, which is still far from being clearly defined, although it is now well established that both peripheral and central mechanisms are involved [2]. Widely recognized guidelines on the neuropathic pain management are being published and updated, setting its pharmacological treatment standards $[3,4]$. Various techniques of interventional pain management have been employed in the past to battle the pain associated with $\mathrm{HZ}$ and PHN, effectiveness of which have been scrutinized and summarized in the 2013 guidelines for interventional pain management of neuropathic pain [5]. According to these guidelines the sympathetic nerve blocks (SNBs) are not recommended in the treatment of PHN. We had been using nerve blocks as the addition to pharmacological treatment in the management of PHN for number of years, which in 2010 was replaced by $5 \%$ lidocaine medicated plasters ( $5 \%$ LMP). Since our overall experience with SNBs had been very plausible, we decided to analyze the available clinical data in order to compare these two methods of PHN therapy.

\section{Material and methods}

The study was approved by institutional ethics committee. We identified and reviewed medical records of 120 consecutive PHN patients who were treated from 2008 until Feb 2014 in the Pain Clinic, Department of Anesthesiology and Intensive Care, Medical Center for Postgraduate Education in Warsaw, and whose clinical picture contained allodynia. PHN was defined as the pain of typical character (constant throbbing pain or intermittent sharp pain, and allodynia), persisting beyond the healing of herpetic skin lesions (more than 4 weeks of the rash onset). Regardless of earlier treatment, the similar regime of management was proposed to all of them, which consisted of either sympathetic nerve block (first 60 patients with allodynia) or $5 \%$ lidocaine medicated plaster (subsequent 60 patients), which if not adequately effective was accompanied first by gabapentinoid, then antidepressant and potent opioid. Our aim was to compare the groups of similar characteristics, thus the number of analyzed cases of PHN treated with SNBs was determined by the number of patients in whom 5\% LMP plasters were used, which until Feb 2014 was 60. For the purpose of unbiased analysis no further cases were added, even if decision was made to exclude the patient.

\subsection{Techniques}

The method of nerve block placement was similar for the given pain location. With the exception of lumbar epidural and sciatic nerve blocks, the local anesthetic used was bupivacaine $2.5 \mathrm{mg} / \mathrm{ml}(0.25 \%)$ with epinephrine $2.5 \mu \mathrm{g} / \mathrm{ml}$. For PHN of the first trigeminal (ophthalmic) nerve its isolated blockade with $5 \mathrm{ml}$ of $0.25 \%$ bupivacaine/epinephrine was implemented. Affected second and/or third trigeminal nerve branch, as well as neck and upper extremity PHN were the indications for stellate ganglion block, for which the modified blind paratracheal approach technique as described by Carron was employed, with $5 \mathrm{ml}$ of $0.25 \%$ bupivacaine/epinephrine [6]. Chest was the most common location of the PHN. Blocks of the corresponding intercostal nerves were placed in these cases with the same concentration of local anesthetic. Three to five neighboring intercostal nerves were typically blocked with $3 \mathrm{ml}$ of local anesthetic solution each, along the scapular line. Sciatic nerve block was implemented in cases where lower extremity was affected. The series of SNBs was started at the first Pain Clinic visit, procedures were repeated twice a week (Mon and Thu). Pharmacological treatment with gabapentin and tricyclic antidepressant was added at the third visit if pain control was not effective, which was defined as Numeric Rating Scale score $>3$ (NRS $>3$ ). If no success was achieved, after two weeks a potent opioid was introduced. SNBs were continued until either a total of 10 blocks were done or success achieved (NRS $\leq 3$ ).

Similarly $5 \%$ lidocaine medicated plasters were prescribed at the first visit, to be applied for $12 \mathrm{~h}$ a day to the affected area, up to 3 plasters at a time, with $12 \mathrm{~h}$ plaster-free time in $24 \mathrm{~h}$. Pain control was reassessed after a week and the same as in nerve blocks group pharmacological treatment was added if found not to be satisfactory (NRS > 3). Unlike SNBs, the lidocaine plasters were continued for the whole period of 8 weeks.

Pain severity after 8 weeks of Pain Clinic care was used to assess the management success or failure. We arbitrarily assumed NRS $<3$ or $=3$ as a cut-off point for therapy success, while spectacular results (NRS 0) were recognized and recorded separately. Statistical analysis was done with Statistica 10 (StatSoft) software: Fisher's exact test was used to compare the rates of treatment results in the groups assessed and student $t$-test to find the difference in mean NRS change between them.

\section{Results}

A total of 120 cases were reviewed. Of 60 patients in each group, 2 were excluded from SNBs group due to the lack of patient's consent to nerve blocking in one case and a history of previously ineffective series of SNBs in the other. Data regarding one case treated with $5 \%$ lidocaine plasters were excluded, as in this case the early onset of significant allergic skin reaction resulted in discontinuation of topical treatment. 
Table 1 - Patients characteristics.

\begin{tabular}{lll} 
& \multicolumn{1}{c}{ SNBS } & 5\% LMP \\
\hline Age (mean \pm SD) & $71.5 \pm 10.8$ & $73.06 \pm 11.1$ \\
Time from HZ in months (median, range) & $2.5(2-36)$ & $2(2-48)$ \\
Male $(n)$ & $15(26 \%)$ & $25(42.4 \%)$ \\
Female $(n)$ & $43(74 \%)$ & $34(57.6 \%)$ \\
Time interval from HZ onset $\leq 3 / 3-12 / \geq 12$ months $(n)$ & $37 / 15 / 6$ & $41 / 11 / 7$ \\
NRS at entry (mean \pm SD) & $8.05 \pm 1.69$ & $7.45 \pm 1.37$ \\
NRS at 8 weeks $($ mean \pm SD) & $2.31 \pm 2.52$ & $2.49 \pm 1.69$ \\
NRS change at 8 weeks (mean \pm SD, $95 \% C I)(p=0.02)$ & $5.88 \pm 2.41 ; 5.24-6.51$ & $5.01 \pm 1.67 ; 4.58-5.45$ \\
Diabetics $(n)$ & 11 & 9 \\
Steroid therapy $(n)$ & 4 & 3 \\
History of malignancy $(n)$ & 11 & 11 \\
Aciclovir in the acute phase of HZ $(n)$ & $45(77.5 \%)$ & $37(62.7 \%)$ \\
Gabapentinoids $(n)$ & $55(94.8 \%)$ & $51(86.4 \%)$ \\
Tricyclic antidepressants $(n)$ & $31(53.4 \%)$ & $42(71.2 \%)$ \\
\hline$n$ - number of patients. & & \\
\hline
\end{tabular}

Their baseline characteristics are given in Table 1. As shown, both groups were similar in terms of their age, male/ female ratio, duration of pain and mean pain severity at the beginning of Pain Clinic care. Areas affected are summarized in Table 2. The review of medical notes have shown that the rate of conditions which are known to affect the immune system and thus facilitate HZ occurrence was not different in both groups assessed (diabetes, steroid therapy, history of malignancy, renal failure). After 8 weeks of treatment in 11 out of 58 patients $(18.9 \%)$ treated with nerve blocks poor treatment results were noted, while in $5 \%$ lidocaine plasters patients the rate of failures was slightly higher, as 16 out of 59 (27.1\%) reported pain severity $>3$ in NRS (Fig. 1). The difference was not statistically significant (two-tailed Fisher's exact test $p=0.38$ ). The rates of pain-free patients $(\mathrm{NRS}=0)$ after 8 weeks of treatment was found to be significantly different: $n=20(34.4 \%)$ in SNBs and $n=8(13.5 \%)$ in $5 \%$ lidocaine group $(p<0.01)$ (Fig. 2). A total of 13 patients has had their symptoms for more than 12 months before the beginning of Pain Clinic treatment: $n=6$ in SNB group and $n=7$ in LMP group. Although most of the pain-free patients in the former group has had their symptoms for no more than 3 months $(n=14)$, five of them for $3-6$ months and one patient for more than a year (18 months). In contrast, all of the 8 patients with NRS $=0$ after 8 weeks of treatment with $5 \%$ lidocaine plasters have had the duration of symptoms for no more than 3 months. When treatment effectiveness was considered as defined by the change in NRS score, 9 patients in both groups reported NRS change of less than 3 points in NRS. The comparison of mean NRS change in the groups assessed is presented in Fig. 3. Mean change in NRS score in SNBs group

Table 2 - Area affected. Data given as number of cases.

\begin{tabular}{lcc} 
& SNBs & 5\% LMP \\
\hline 1st branch of trigeminal nerve & 9 & 2 \\
Head and neck (2nd and 3rd & 3 & 10 \\
$\quad$ branch of trigeminal nerve) & & \\
Upper extremity & 10 & 5 \\
Thorax & 32 & 38 \\
Lower extremity & 4 & 4 \\
\hline
\end{tabular}

was significantly greater than that of 5\% LMP group (5.88 \pm 2.41 , 95\% CI $5.24-6.51$ vs. $5.01 \pm 1.67,95 \%$ CI $4.58-5.45$, $p=0.02$ ). Of pain-free patients at 8 weeks (NRS $=0$ ), 9 out of 20 in SNBs group had their pain severity at NRS 9 (2 patients) and NRS 10 (7 patients) at the study entry. Only one of NRS =0 patients after LMP treatment have started the Pain Clinic treatment with NRS $=9$, none with NRS $=10$. Apart from one case of major allergic reaction to lidocaine plaster, no other relevant side effects of the nerve blocks and lidocaine plasters were noted. In one LMP case some transient redness was reported and described as being "annoying", but did not result in discontinuation of the treatment. Four patients in SNBs and one in LMP group complained of mild vertigo after gabapentin was introduced, which resolved after it was changed to pregabalin.

\section{Discussion}

This analysis presents the outcomes of PHN management with two treatment strategies, of which one is validated by recently published guidelines and the other might be considered conservative, as its use was never supported by the results of randomized controlled trials which would allow some

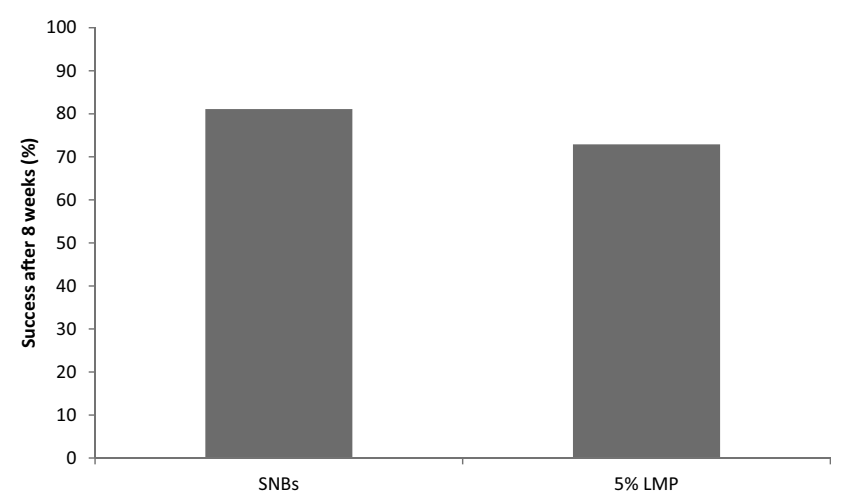

Fig. 1 - Success rate (NRS $<3$ or $=3$ ) after 8 weeks of treatment $(p=0.38)$. 


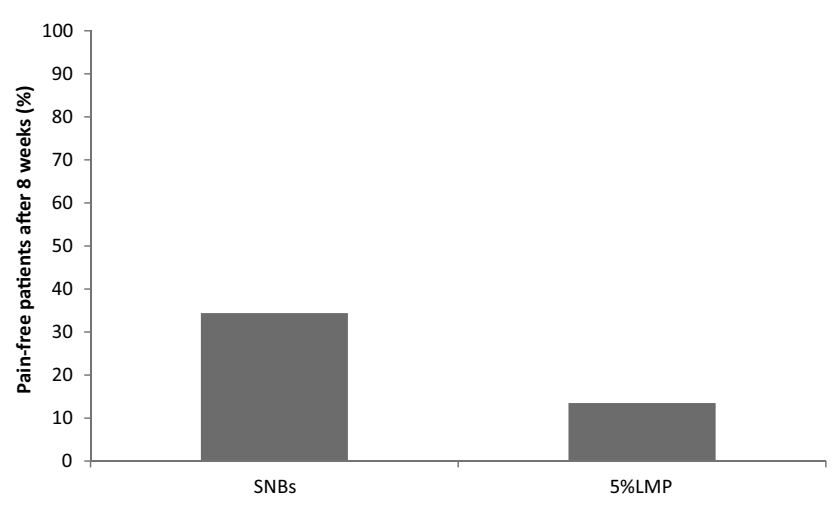

Fig. 2 - Pain-free patients (NRS = 0) after 8 weeks of treatment $(p<0.01)$.

meaningful conclusions to be drawn from. Both modes of action are directed against peripheral component of neuropathic pain - when the damage of afferent nociceptors results in marked alodynia. Mechanisms by which sympathetic system might be involved in neuropathic pain pathogenesis are thought to include direct, pathological links between somatic afferent and postganglionic autonomic nerves that stem from abnormal nerve fibers sprouting in the area of neuronal damage [7], and may be mediated by noradrenaline [8]. The SNBs use for HZ - related pain dates back to as early as 1938, when Rosenak reported procaine use in the treatment of the acute phase of HZ [9]. Since then two more indications for nerve blocks in conditions related to VZV infection have been described-prevention of PHN and pain relief in established PHN, all of which have recently come under scrutiny. Pathomechanism of its beneficial effect appears not to be well understood and yet clarified. Although some reasonable rationale behind sympathetic blockade within the course of $\mathrm{HZ}$ has been reported, it does not provide explanation for its benefit in already established neuralgia [10]. Literature data regarding the efficacy of nerve blocks in the acute phase of $\mathrm{HZ}$ is much more prominent than data in support of its use in PHN management $[10,11]$.

After early reports by Colding [12,13], clinical reports were limited to retrospective analyses, case series and case reports, which suggested beneficial but mostly short-lived effect of SNBs in cases where routine approach was not effective.

Although used by many pain specialists, nerve blocking in PHN have not earned enough credit to become a recommended strategy in most recently published guidelines for

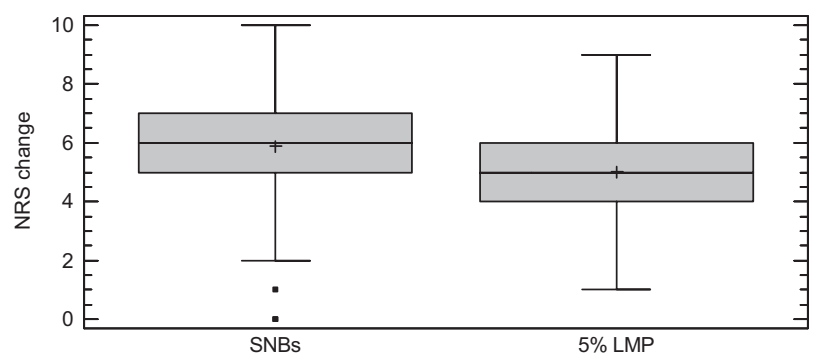

Fig. 3 - NRS change in the groups assessed $(p=0.02)$. interventional management of neuropathic pain [5]. Quite the contrary-it is recommended not to use them for PHN. Our long standing experience with nerve blocks and PHN was plausible, but introduction of topical lidocaine in the form of 5\% LMP and subsequent recommendations for neuropathic pain management have brought an end to its use.

Topical preparations of lidocaine on the other hand, being superficially active with only minimal systemic absorption, have proved to be helpful in reducing peripheral neuropathic pain symptoms, including PHN. They are deprived of relevant side effects associated with the use of potent systemic drugs used for neuropathic pain management, like dizziness and somnolence. The efficacy of $5 \%$ lidocaine plasters for PHN management have been assessed in randomized, controlled trials, as well as in open-label trials, most of which were subsequently summarized in meta-analyses, where their usefulness was confirmed $[14,15]$. In a systematic review of 5\% LMP vs. other treatments for PHN Wolff et al. have identified two trials comparing the efficacy of 5\% LMP vs. placebo and one assessing LMP vs. pregabalin [15]. Lidocaine plasters were found to be more effective than placebo. Pregabalin at the titrated doses was found to be less effective after 4 weeks of treatment than LMP when the success was defined as the mean change in NRS $\geq 2$ or an absolute NRS $<4$ or $=4$. Nevertheless, after thorough analysis of available data on 5\% LMP vs. placebo and other modes of treatment, it was concluded that further research in this area is warranted to allow for greater number of cases to be included in systematic reviews and analyses, as the number of good quality studies is still small. Also, there is a marked number of limitations reported that may affect their value. In the study by Binder et al. [16], where LMP was reported to be more efficacious than placebo, the concomitant therapy was allowed, taken by $60 \%$ of patients who have originally entered the study, and described as being mostly anticonvulsants, while more specific description is lacking [16]. Similarly, the time from the symptoms onset is usually difficult to standardize.

Although good clinical effect of PHN management was equally frequent in our LMP and SNBs patients, the rate of spectacular results in SNBs group was significantly higher. It appears that in some patients peripheral nerve blocking resulting in the interruption of sympathetic signals to the affected area may effect in more spectacular pain relief than that provided by topically applied lidocaine. The fact that the duration of pain in most of the pain-free patients in SNBs group and none in LMP was more than 3 months may point to the vascular nature of the pathophysiological background behind this phenomenon. Considering the theory by Winnie and Hartwell [10], it may be hypothesized that long-lasting ischemia leads to irreversible damage to cutaneous nerve endings, which are therefore less susceptible to superficially active lidocaine preparations, while SNBs' target is located more proximally and effects in decreased noradrenaline release from sympathetic nerve endings. Compliant with the theory of noradrenaline-mediated link between postganglionic sympathetic fibers and pain afferents (chemical coupling and/or abnormal $\alpha$-adrenoreceptors activation in PHN pain), this should decrease the PHN pain $[7,8]$. This theory could obviously be challenged by data pointing to some other than sodium channel blocking actions of topical lidocaine in 
neuropathic pain, as it appears to be effective in cases where the skin is deprived of nociceptors [17]. Nevertheless, the difference in spectacular results is significant. It may be argued that our results are in contrast with widely cited early reports by Colding, dated back to nineteen sixties and seventies, where SNBs provided significant but short-lived PHN pain relief $[12,13]$. However, it has to be kept in mind that the repertoire of pharmacological therapy was very limited at the time these reports were published. In current studies most of PHN patients take at least one of the drugs with proven effectiveness in neuropathic pain. Almost $95 \%$ of our patients in SNBs and $86 \%$ in 5\% LMP group have taken antiepileptics, in many cases tricyclic antidepressants were added as well. It should also be stated that occasionally published case reports, where SNBs proved to be effective after other treatments failed to provide sufficient pain control, may be in support of our findings $[18,19]$. Considering their therapeutic effectiveness, our results suggest that despite previous criticism sympathetic (stellate ganglion) block as well as peripheral nerve (i.e. sciatic nerve, intercostal nerve) block might be of clinical value and, as such, still constitute options worth considering in allodynia-affected PHN cases.

\section{Conflict of interest}

None declared.

\section{Acknowledgement and financial support}

None declared.

\section{Ethics}

The work described in this article has been carried out in accordance with The Code of Ethics of the World Medical Association (Declaration of Helsinki) for experiments involving humans; Uniform Requirements for manuscripts submitted to Biomedical journals.

\section{REFERENCES}

[1] Niv D, Maltsman-Tseikhin A, Lang E. Postherpetic neuralgia: what do we know and where are we heading? Pain Physician 2004;7(2):239-47.

[2] Argoff CE, Katz N, Backonja M. Treatment of postherpetic neuralgia: a review of therapeutic options. J Pain Symptom Manage 2004;28(4):396-411.

[3] American Society of Anesthesiologists Task Force on Chronic Pain Management; American Society of Regional Anesthesia and Pain Medicine. Practice guidelines for chronic pain management: an updated report by the
American Society of Anesthesiologists Task Force on Chronic Pain Management and the American Society of Regional Anesthesia and Pain Medicine. Anesthesiology 2010;112(4):810-33.

[4] Attal N, Cruccu G, Baron R, Haanpää M, Hansson P, Jensen $\mathrm{TS}<\mathrm{ET}-\mathrm{Al}>$. European Federation of Neurological Societies. EFNS guidelines on the pharmacological treatment of neuropathic pain: 2010 revision. Eur J Neurol 2010;17(9): 1113-23.

[5] Dworkin RH, O'Connor AB, Kent J, Mackey SC, Raja SN, Stacey BR, et al. International Association for the Study of Pain Neuropathic Pain Special Interest Group. Interventional management of neuropathic pain: NeuPSIG recommendations. Pain 2013;154(11):2249-61.

[6] Carron H, Litwiller R. Stellate ganglion block. Anesth Analg 1975;54(5):567-70.

[7] Jänig W, Levine JD, Michaelis M. Interactions of sympathetic and primary afferent neurons following nerve injury and tissue trauma. Prog Brain Res 1996;113:161-84.

[8] Sato J, Perl ER. Adrenergic excitation of cutaneous pain receptors induced by peripheral nerve injury. Science 1991;251(5001):1608-10.

[9] Rosenak S. Procaine injection treatment of herpes zoster. Lancet 1938;1056-8.

[10] Winnie AP, Hartwell PW. Relationship between time of treatment of acute herpes zoster with sympathetic blockade and prevention of post-herpetic neuralgia: clinical support for a new theory of the mechanism by which sympathetic blockade provides therapeutic benefit. Reg Anesth 1993;18(5):277-82.

[11] Rickles JA. Ambulatory use of sympathetic nerve blocks: present day clinical indications. Angiology 1977;28(6): 394-402.

[12] Colding A. The effect of regional sympathetic blocks in the treatment of herpes zoster: a survey of 300 cases. Acta Anaesthesiol Scand 1969;13:133-41.

[13] Colding A. Treatment of pain: organization of a pain clinic: treatment of acute herpes zoster. Proc R Soc Med 1973;66:541-3.

[14] Garnock-Jones KP, Keating GM. Lidocaine 5\% medicated plaster. A review of its use in postherpetic neuralgia. Drugs 2009;69(15):2149-65.

[15] Wolff RF, Bala MM, Westwood M, Kessels AG, Jleijnen K. 5\% lidocaine-medicated plaster vs other relevant interventions and placebo for post-herpetic neuralgia (PHN): a systematic review. Acta Neurol Scand 2011;123(5):295-309.

[16] Binder A, Bruxelle J, Rogers P, Hans G, Bösl I, Baron R. Topical 5\% lidocaine (lignocaine) medicated plaster treatment for post-herpetic neuralgia: results of a double blind, placebo-controlled, multinational efficacy and safety trial. Clin Drug Investig 2009;29(6):393-408.

[17] Herrmann DN, Pannoni V, Barbano RL, Penjnella-Vaughan J, Dworkin RH. Skin biopsy and quantitative sensory testing do not predict response to lidocaine patch in painful neuropathies. Muscle Nerve 2006;33(1):42-8.

[18] Gomes RT, de Nazareth Pedras RB, da Silva JF, de Aguiar MC. Sympathetic nerve blocks in mandibular herpes zoster and postherpetic neuralgia. Headache 2007;47(5):728-30.

[19] Peterson RC, Patel L, Cubert K, Gulati A. Serial stellate ganglion blocks for intractable postherpetic itching in a pediatric patient: a case report. Pain Physician 2009;12(3): 629-32. 